
\title{
25 Research Square \\ SLCO and SLC22 Solute Carriers: Establishing Differential Expression Patterns in Human Breast Cancer Cell Lines and Tumour Samples
}

\section{Rachel Sutherland}

Newcastle University Institute of Genetic Medicine

\section{Annette Meeson}

Newcastle University Institute of Genetic Medicine

Simon Lowes ( $\square$ simon.lowes@doctors.org.uk)

Newcastle University Institute of Genetic Medicine https://orcid.org/0000-0003-1959-266X

\section{Research article}

Keywords: Breast cancer, SLCO, SLC22, Drug transport, Chemotherapeutic drugs, Estrogen transport

Posted Date: March 30th, 2021

DOI: https://doi.org/10.21203/rs.3.rs-349903/v1

License: (c) (i) This work is licensed under a Creative Commons Attribution 4.0 International License.

Read Full License 


\section{Abstract}

Background: The uptake of nutrients essential for cell growth and survival is facilitated by solute carrier (SLC) transporters. The SLCO and SLC22 subfamilies mediate the uptake of substrates relevant to breast cancer (BC), including steroid hormones and anticancer drugs, and accumulating evidence suggests that altered expression of these transporters may affect BC pathogenesis by influencing cell proliferation and anticancer drug resistance.

Methods: The differential expression of 11 SLCO and 14 SLC22 transporters was investigated using semiquantitative and quantitative PCR in MCF-7 and MDA-MB-231 BC cell lines and in human BC tissue samples.

Results: 8 SLCO and 10 SLC22 transporters were expressed in at least one cell line. Of these SLC01B1, SLC01B3, SLC03A1, SLC22A1, SLC22A3, SLC22A4 and SLC22A16 showed higher expression in MDA-MB231 than MCF-7 cells. Conversely, SLCO2A1, SLC04A1, SLC04C1, SLC05A1, SLC22A5 showed higher expression in MCF-7 than MDA-MB-231 cells. In four human breast cancer samples, there was variable expression of the 25 SLC transporters, however, 11 SLCO and 9 SLC22 transporters were detected in at least one sample.

Conclusion: BC cells express a variety of SLCs capable of transporting a range of steroid hormones and clinically relevant anticancer drugs, which could implicate them in BC pathogenesis and anti-cancer drug resistance.

\section{Background}

Mechanisms that underpin the pathogenesis of $\mathrm{BC}$ are highly complex, and gaining insights into these are further complicated by the heterogeneity between breast tumours, a factor that is recognised to contribute to treatment failure (1). One mechanism that may contribute directly to disease pathogenesis and the effectiveness of therapy is the balance between uptake and efflux transporters, which facilitate the bi-directional transport of solutes into and out of cells. ABC transport proteins such as ABCB1, ABCG2 and $A B C C 1$, mediate the efflux of chemotherapeutic agents out of cells, which has established them as important mediators of $\mathrm{BC}$ progression and treatment (2), and can result in multidrug resistance (MDR), particularly when they are overexpressed (3-6).

In contrast to the efflux transporters, comparatively little is known about the expression and role of transporters mediating uptake of substrates into BC cells. Of likely significance are members of the solute carrier (SLC) superfamily of transporters. The SLC superfamily is the second largest group of membrane proteins in humans, comprising over 400 proteins arranged into 65 subfamilies. They all share the same basic structure, a 12 transmembrane domain with intracellular termini $(7,8)$. Physiologically, they contribute to homeostasis by facilitating the uptake of a variety of endogenous and exogenous anionic, cationic and zwitterionic substrates into cells using electrochemical and concentration gradients $(9,10)$. 
The range of substrates for SLC transporters is diverse, and many members within the same family share overlapping substrate specificity $(9,11,12)$.

The SLCO subfamily, which incorporates the organic anion transporting peptides (OATP), and the SLC22 subfamily, which incorporates the organic anion (OAT), organic cation (OCT) and organic carnitine/zwitterion (OCTN) transporters, are of particular interest in $\mathrm{BC}$, as many of their members transport substrates important for $\mathrm{BC}$ progression and treatment, including estrogens and anticancer drugs $(9,12-18)$.

The majority of newly diagnosed BCs are hormone responsive (19), and steroid hormones promote carcinogenesis by inducing cellular proliferation through their receptor-mediated hormonal activity. Routine diagnostic workup of $\mathrm{BC}$ samples typically includes testing for the expression of estrogen receptor (ER), progesterone receptor (PR) and human epidermal growth factor receptor 2 (HER2), which can help guide treatment decisions and prognosis. Tumours that lack expression of all three receptors are termed 'triple negative' (ER', $\left.\mathrm{PR}^{-}, \mathrm{HER}{ }^{-}\right)$and have a poorer prognosis (20). Estrogens are substrates for 8 of the 11 human SLCO (OATP) transporters (SLC01A2/OATP1A2, SLC01B1/OATP1B1, SLC01B3/OATP1B3, SLC01C1/OATP1C1, SLCO2B1/OATP2B1, SLC03A1/OATP3A1, SLC04A1/OATP4A1 and SLC04C1/OATP4C1) and 6 of 14 functionally characterised SLC22 transporters (SLC22A3/OCT3, SLC22A7/OAT2, SLC22A8/OAT3, SLC22A9/OAT7, SLC22A10/OAT5, SLC22A11/OAT4) $(8,9,12,15,18$, 21-23).

Although the expression of a few select SLC transporters in various BC cell lines and tissues has been demonstrated, the available evidence is piecemeal. This study aimed to provide a comprehensive overview of SLCO and SLC22 expression in hormone responsive (MCF-7) and hormone non-responsive (MDA-MB-231) BC cell lines and in human BC tissue samples, allowing an analysis of differential expression patterns.

\section{Methods}

\section{Human tissue samples}

Fresh breast tumour samples were obtained at the Breast Screening and Assessment Unit, Queen Elizabeth Hospital, Gateshead, UK from women with biopsy-proven BC who were deemed clinically unsuitable for surgery and who were instead undergoing ultrasound-guided vacuum-assisted tumour excision as part of a separate clinical research study (REC number 18/NW/0168). As part of the study, all women gave written consent for samples of their tissue to be used in this project. Breast tumour samples were placed directly into RNA-later from a cohort of four patients aged 78-82 years. All tumours were invasive ductal carcinoma, $\mathrm{ER}^{+} \mathrm{HER} 2^{-}$. All patients had received some form of primary endocrine therapy prior to tumour excision.

\section{Cell Lines and Culture}


BC cell lines used were MCF-7 (purchased from ECACC, Salisbury, UK) and MDA-MB-231 (provided by MD Anderson, Houston, Texas, USA). Both cell lines were validated via STR Profiling using PowerPlex $\AA_{16}$ HS PCR Amplification Kit (Promega, Wisconsin, USA) according to the manufacturer's instructions. The MCF7 and MDA-MB-231 cells were cultured as described previously (24).

\section{RNA Isolation}

Total RNA was isolated from cell lines using the RNeasy ${ }^{\circledR}$ Micro Kit (Qiagen, Manchester, UK) and from patient tumour samples using TRI Reagent ${ }^{\circledR}$ (Merck, USA) followed by the RNeasy ${ }^{\circledR}$ Micro Kit (Qiagen, Manchester, UK). All protocols were performed as per manufacturers' instructions.

\section{Positive Controls}

Human total kidney RNA was purchased from Thermo Fisher Scientific (Massachusetts, USA). Human brain RNA was isolated from tissue from the Parkinson's UK Brain Bank, UK and kindly gifted by Dr llse Pienaar, Sussex University, UK. Human placental tissue was provided by the Newcastle Uteroplacental Tissue Bank (REC 16/NE/0167), Newcastle University, UK. Human heart tissue was provided by the Newcastle Institute of Transplantation Tissue Biobank, UK, ethics agreement number IOT028. The human liver cancer cell line, HepG2 was kindly gifted by Professor Matthew Wright, Newcastle University, UK. The human lung squamous cell carcinoma cell line, $\mathrm{NCl}-\mathrm{H} 520$, was kindly gifted by Dr Alastair Greystoke, Newcastle University, UK. RNA was isolated from placental and heart tissue using TRI Reagent ${ }^{\circledR}$ (Merck, USA). RNA was isolated from HepG2 and NCl-H520 cells using the RNeasy ${ }^{\circledR}$ Mini Kit (Qiagen, Manchester, UK).

\section{cDNA Synthesis}

cDNA was synthesised using the Bioline cDNA Synthesis Kit (Scientific Laboratory Supplies, Nottingham, UK) for cell lines and patient-derived tumour samples as per the manufacturer's instructions.

\section{Semi-quantitative Polymerase Chain Reaction (sq-PCR)}

Sq-PCR was performed as described previously (25). Details of primer sequences and reaction conditions are listed in Additional file 1. All PCR reactions included GAPDH loading controls and positive/nontemplate negative controls.

\section{Quantitative Real-Time Polymerase Chain Reaction (qPCR)}

qPCR experiments were performed as described previously (26). The reaction was set up at $95^{\circ} \mathrm{C}$ for 10 mins followed by 40 cycles of $95^{\circ} \mathrm{C}$ for $15 \mathrm{~s}$ and $60^{\circ} \mathrm{C}$ for 1 mins. Relative mRNA expression was calculated using the comparative $\Delta \Delta \mathrm{C}_{\mathrm{t}}$-method. TaqMan probes were purchased from Applied Biosystems (California, USA). Assay IDs for each target are listed in Additional file 1.

\section{Statistical and Data Analysis}

Statistical analysis was performed using IMB SPSS Statistics 24 (Chicago, Illinois, USA) and graphs were generated on GraphPad Prism version 8 (GraphPad Software, San Diego, USA). All experiments were 
performed in triplicate for cell lines pertaining to three different cell passages. Results are presented as mean \pm SD. All statistical analyses were performed on $\log _{2}$ transformed data. Statistical significance was determined by one-sample t-test or independent samples t-test as appropriate. All results are reported as significant at $p<0.05$.

\section{Results}

\section{SLCO and SLC22 transporter expression in MCF-7 and MDA- MB-231 cell lines SLCO subfamily}

Differential mRNA expression of 11 human SLCO genes was compared between the MCF-7 and MDA-MB231 cells using sq-PCR (Fig. 1A). Three of the genes, SLC01C1/OATP1C1, SLCO2B1/OATP2B1 and SLC06A1/OATP6A1, showed no/very low expression in both cell lines. Of the genes that did show expression in either cell line, SLC01B1/OATP1B1 and SLC01B3/OATP1B3 were highly expressed in MDAMB-231 but showed no or low expression in MCF-7 cells. Conversely, SLC04C1/OATP4C1, SLC05A1/OATP5A1 and SLC02A1/OATP2A1 showed higher expression in MCF-7 compared to MDA-MB231 cells. The remaining transporters, SLC01A2/OATP1A2, SLC04A1/OATP4A1 and SLCO3A1/OATP3A1, showed similar expression between both cell lines (Fig. 1A). Positive controls are listed in Additional file 1 .

qPCR was performed for the 11 SLCO transporter genes in both cell lines. Of these, 3 genes were below the detection limit for both cell lines: SLC01A2/OATP1A2, SLC01C1/OATP1C1, and SLC06A1/OATP6A1 (data not shown). Both SLC01B1/OATP1B1 and SLC01B3/OATP1B3 showed considerably higher expression in MDA-MB-231 cells compared to the calibrator sample (34-fold $(p=0.013)$ and 1418-fold ( $p$ $=0.001$ ) higher, respectively), while in MCF-7 cells, SLC01B1/OATP1B1 expression was detectable but lower than that of the calibrator $(p=0.423)$, whereas SLC01B3/OATP1B3 was below the detection limit (Fig. 1B).

The remaining transporters all showed lower expression in both cell lines compared to the calibrator. However comparison of the fold change of the two cell lines relative to the calibrator suggests that SLC02A1/OATP2A1 and SLC05A1/OATP5A1 $(p=<0.0001)$ (Fig. 1) are expressed at a higher level in the MCF-7 than the MDA-MB-231 cells (P value for SLCO2A1 not stated as MDA-MB-231 values all below the detection limit). Conversely, no significant difference in the expression of SLC04A1/OATP4A1 $(p=0.078)$, SLC04C1/OATP4C1 $(p=0.222)$, SLC02B1/OATP2B1 $(p=0.831)$ and SLC03A1/OATP3A1 $(p=0.661)$ was detected between the cell lines (Fig. 1B). Low SLCO2B1 expression was detected in both cell lines using qPCR where it had not been identified by sq-PCR.

\section{SLC22 subfamily}


Of 14 SLC22 genes investigated using sq-PCR, 6 were found to have no expression in either cell line: SLC22A2/OCT2, SLC22A7/OAT2, SLC22A9/OAT7, SLC22A10/OAT5, SLC22A11/OAT4 and SLC22A13/OAT10 (Fig. 2A). The remaining transporter genes showed variable expression between the two cell lines. SLC22A3/OCT3, SLC22A4/OCTN1, SLC22A8/OAT3, and SLC22A12/URAT1 showed higher expression in MDA-MB-231 cells (Fig. 2A), and the remaining transporters, SLC22A1/OAT1, SLC22A5/OCTN2, SLC22A6/OAT1 and SLC22A16/OCT6, showed similar expression levels in both cell lines (Fig. 2A).

When qPCR analysis was carried out, 7 of 14 SLC22 transporters, SLC22A2/OCT2, SLC22A6/OAT1, SLC22A7/OAT2, SLC22A8/OAT3, SLC22A9/OAT7, SLC22A10/OAT5, and SLC22A12/URAT1, were below the detection limit in both cell lines (data not shown). SLC22A3/OCT3 showed a similar level of expression in MDA-MB-231 as the calibrator, but expression was below the detection limit in MCF-7 cells (Fig. 2B). SLC22A1/OCT1, SLC22A4/OCTN1, SLC22A5/OCTN2, SLC22A11/OAT4, SLC22A13/OAT10 and SLC22A16/OCT6 all showed lower expression in the two cell lines relative to the calibrator. Direct comparison between the cell lines showed that SLC22A1/OCT1, SLC22A4/OCTN1, SLC22A13/OAT10, and SLC22A16/0CT6 showed higher expression in the MDA-MB-231 cells (all $p<0.05$ ) (Fig. 2B), whereas SLC22A5/OCTN2 showed higher expression in MCF-7 cells $(p=0.001)$ (Fig. 2B). The expression of SLC22A11/OAT4 was not significantly different between the cell lines $(p=0.240)$.

\section{Expression of SLC transporters in human breast cancer tissue samples}

Expression of SLC transporters was then investigated in tumour samples from patients with biopsyproven invasive ductal carcinomas.

\section{SLCO subfamily}

The same 11 SLCO transporters were investigated as for the cell lines (Fig. 3). Using sq-PCR, the overall expression pattern of the SLCO subtypes was similar across each of the patient samples, but there was variability of expression levels between patients. SLC01A2/OATP1A2, SLC01C1/OATP1C1, SLCO2A1/OATP2A1, SLCO2B1/OATP2B1, and SLC03A1/OATP3A1 were consistently expressed in all patients, with patient 1 showing a greater band intensity of both SLC01C1/OATP1C1 and SLC02A1/OATP2A1. Expression of SLC01B3/OATP1B3 was variable, with the highest levels seen in patient 2. There was no/negligible expression of SLC01B1/OATP1B1, SLCO4A1/OATP4A1, SLC04C1/OATP4C1, or SLC05A1/OATP5A1 in any of the patients, with weak expression of SLC06A1/OATP6A1 (Fig. 3).

qPCR was carried out for further analysis. Because of the very small quantities of tissue available, analysis was limited to 8 of the 11 transporters, selected based on expression in the cell lines and their likely clinical relevance in relation to their known substrate specificities (Fig. 4). SLC01A2/OATP1A2 was expressed in all tumour samples with highest fold change in patient 3 (20.66-fold) and the lowest in patient 2 (0.37-fold) relative to the control (Fig. 4). SLC01C1/OATP1C1, SLCO2A1/OATP2A1 and 
SLC04C1/OATP4C1 showed low expression in all four tumours, as was previously identified via sq-PCR. The expression of SLCO2B1/OATP2B1 was higher in all four tumours relative to the control (2.92-5.64fold); while sq-PCR also detected high expression of this transporter. SLC03A1/OATP3A1 expression was 1.75-2.02-fold higher than controls in tumours 1-3, but lower than the control in patient 4 . The expression of SLC01B1/OATP1B1 and SLC01B3/OATP1B3 showed a high degree of variability between patients. SLC01B1/OATP1B1 was expressed 4-fold higher than the control in patient 1, 88-fold higher in patient 4, 1,446-fold higher in patient 2, and 1,152-fold higher in patient 3. SLCO1B3, on the other hand, was below the detection limit in patients 1 and 2, and 53- and 57-fold higher than the calibrator in patients 3 and 4 (Fig. 4).

\section{SLC22 subfamily}

14 SLC22 transporters were analysed via sq-PCR in the tumour samples. As was observed for the SLCO transporters, the pattern of expression was largely consistent between the four patients. SLC22A5/OCTN2 and SLC22A16/OCT6 were both highly expressed and showed a similar degree of expression across all four tumours (Fig. 5); SLC22A5/OCTN2 appeared to show similar expression levels to the positive control, whereas SLC22A16 (OCT6) was much more highly expressed in the tumours than in the control. SLC22A1/OCT1 and SLC22A9/OAT7 were likewise expressed in all tumours however the degree of their expression was variable (Fig. 5). SLC22A2/OCT2 and SLC22A4/OCTN1 showed variability between patients. SLC22A2/OCT2 showed expression in patients 1 and 2 but appeared not be expressed in patients 3 and 4 . Conversely, SLC22A4/OCTN1 showed low/no, expression in patients 1 and 2 but expression equivalent to the positive control in patients 3 and 4 (Fig. 5). SLC22A3/OCT3 showed lowmedium expression in all patient tumours that was equivalent to the expression shown in the positive control (placental RNA). SLC22A10/OAT5 showed low expression in all tumours except patient 3 where the expression appeared to be slightly higher. SLC22A6/OAT1 showed low expression in patients 1,3 , and 4. SLC22A7/OAT2, SLC22A8/OAT3, SLC22A11/OAT4, SLC22A12/URAT1 and SLC22A13/OAT10 were not expressed in any of the tumours (Fig. 5).

qPCR was then used to quantify the expression of 6 of the 11 SLC22 transporters; again, the small amounts of human tissue necessitated a more limited analysis. Although the sq-PCR results suggested that SLC22A2/OCT2 may be expressed at low levels in at least two of the tumours (patients 1 and 2), qPCR analysis detected no SLC22A2/OCT2 expression in any of the tumours (Fig. 6). SLC22A7/OAT2 and SLC22A8/OAT3 were likewise not expressed in the tumour samples, consistent with sq-PCR data. Very low expression of SLC22A11/OAT4 was detected compared to the control, in contrast to sq-PCR, where no expression was detected. SLC22A3/OCT3 and SLC22A1/OCT1 both showed higher expression in the tumours compared to the controls, however levels were variable between patients (1.38-22.04 fold and 1.14-10.34 fold, respectively); both had also been detected using sq-PCR (Fig. 6).

\section{Discussion}

This study represents the most comprehensive analysis of different SLC subfamily members in breast cancer tissues to date. As well as consolidating some of the existing piecemeal data it characterises the 
expression of other SLC members not previously investigated in breast cancer tissues.

In relation to the SLCO transporters, the findings from the present study, together with two previous smaller studies $(19,27)$, now allow certain commonalities in the expression patterns in MCF-7 and MDAMB-231 cells to be mapped out. SLC01B1/OATP1B1 and SLC01B3/OATP1B3 are consistently highly expressed MDA-MB-231 cells, with little or no expression in MCF-7 cells. SLCO2A1/OATP2A1, SLC04A1/OATP4A1, SLC05A1/OATP5A1, and SLC04C1/OATP4C1 are consistently expressed in both cell lines, but higher in MCF-7 cells. SLCO3A1/OATP3A1 is consistently expressed in both cell types but is not consistently higher in one cell type versus the other. SLC01C1/OATP1C1 and SLCO6A1/OATP6A1 are consistently not expressed in either cell line. SLC01A2/OATP1A2 and SLCO2B1/OATP2B1 show variable and inconsistent expression patterns in both cell lines across the three studies, as well as two other additional studies $(19,27-29)$.

In a similar vein to the SLCOs, there are relatively few studies investigating SLC22 expression in breast cancer cells. The finding in this study were that SLC22A2/OCT2, SLC22A7/OAT2, SLC22A9/OAT7 and SLC22A10/OAT5 showed no expression in either MCF-7 or MDA-MB-231 cells. Although a previous study of the NCl-60 cell line panel corroborated the present study's findings for SLC22A7/OAT2 and SLC22A9/OAT7, with no expression detected in either cell line, the data for SLC22A2/OCT2 are at odds with another study, which found that it was relatively highly expressed in MDA-MB-231 cells and minimally expressed in MCF-7 cells (30). Similarly, although SLC22A6/OAT1, SLC22A8/OAT3 and SLC22A12/URAT1 showed mixed expression in the two cell lines based on sq-PCR, all were undetectable on GPCR. The reason for this discrepancy is unclear, but the GPCR findings are again consistent with expression data from the $\mathrm{NCl}-60$ cell line panel, which showed that all three were undetectable in either cell line (31). SLC22A11/OAT4 and SLC22A13/OAT10 were both detected using qPCR, but showed very low expression levels, again consistent with the $\mathrm{NCl}-60$ panel data (31).

Finally, SLC22A1/OCT1, SLC22A3/OCT3, SLC22A4/OCTN1, SLC22A5/OCTN2 and SLC22A16/OCT6 were all detected in both cell lines, consistent with previous findings (31) and, with the exception of SLC22A5/OCTN2, expression levels higher in MDA-MB-231 compared to MCF-7 cells. The observation that SLC22A1/OCT1 and SLC22A3/OCT3 show higher expression in MDA-MB-231 versus MCF-7 cells reflects that of a previous study (30). Likewise the pattern of SLC22A5/OCTN2 expression is also consistent with that of previous studies, which demonstrated higher expression in the MCF-7 cells (31, 32). Studies have also revealed that SLC22A5/OCTN2 is significantly overexpressed in $\mathrm{ER}^{+}$cell lines versus $\mathrm{ER}^{-}$cell lines (32).

Perhaps surprisingly, the transporter profiles of the four human tumour samples, based on sq-PCR, and where available, qPCR data, showed more consistency between one another than with the cell lines. The SLCO genes consistently present in all tumour samples were SLC01C1/OATP1C1, SLC01A2/OATP1A2, SLC02B1/OATP2B1, SLC03A1/OATP3A1, SLC01B1/OATP1B1, SLC04C1/OATP4C1, SLC02A1/OATP2A1, and SLC06A1/OATP6A1. All these transport steroid hormones and a range of chemotherapeutic agents, except for SLC02A1/OATP2A1, a prostaglandin transporter, and SLC06A1/OATP6A1, which is not 
currently well characterised. The pattern of expression is noteworthy since SLC01C1/OATP1C1, SLC01A2/OATP1A2, SLC02B1/OATP2B1, and SLC06A1/OATP6A1 showed either no or inconsistent expression in the cell lines.

SLC01B3/OATP1B3 was expressed at high levels in the two of the tumour samples but was not detected in the other two samples. Again, SLC01B3/OATP1B3 transports a range of steroid hormones and clinically relevant chemotherapeutic agents $(18,31,33-35)$, so could be important in mediating hormone and/or uptake in some tumours. Whereas the reason for the clear differential expression pattern in two of the four tumours is unclear at this stage, it may simply reflect disease heterogeneity, with previous studies having either shown no detectable expression in breast tumours $(27,36-38)$, or, in one study, expression in 50\% of tissues (39). Similarly the observation that SLC01B1/OATP1B1 and SLC01B3/OATP1B3 demonstrated such high expression in MDA-MB-231 cells and little or no expression in MCF-7 cells seems somewhat paradoxical given their role in estrogen transport and the fact that MDA-MB-231 cells are classically hormone non-responsive, but in this case the high expression of these transporters could indicate a potentially significant role in the uptake of therapeutic drugs. SLCO4A1/OATP4A1 and SLC05A1/OATP5A1 were not expressed in any of the tumour samples, despite being expressed in both cell lines, greater in MCF-7. The positive control used for SLC05A1 was CDNA from a lung cancer cell line (NCI-H520). Several positive controls were tested for SLC05A1 with only lung CDNA showing low but detectable expression in agreement with previous findings (40). This, combined with previous studies, suggests these transporters are unlikely to be of functional significance in breast cancer; although SLC04A1/OATP4A1 transports steroid hormones, SLC05A1/OATP5A1 remains to be characterised, and previous studies suggest that expression levels of both transporters in non-malignant breast tissue is either the same or even higher than that in breast cancer tissue $(27,36)$.

Of the SLC22 genes, SLC22A1/OCT1, SLC22A3/OCT3, SLC22A16/OCT6, SLC22A5/OCTN2, SLC22A6/OAT1, SLC22A10/OAT5 and SLC22A9/OAT7 were present in all tumour samples. Of these, all were expressed in the cell lines except SLC22A10/OAT5 and SLC22A9/OAT7, which were expressed in neither cell line. SLC22A2/OCT2, SLC22A11/OAT4, and SLC22A4/OCTN1 were also present in the tumours but their expression was variable across the different samples. SLC22A2/OCT2 was not present in either cell line, whereas SLC22A11/OAT4 and SLC22A4/OCTN1 showed expression in both cell lines. These SLC22 genes are mainly transporters of organic cations and anticancer drugs, and, in the case of SLC22A9/OAT7, estrone-3-sulfate (E-3-S), so any of these could have potential significance in the treatment or pathogenesis of breast cancer.

SLC22A7/OAT2, SLC22A8/OAT3, SLC22A13/OAT10, and SLC22A12/URAT1 were not expressed in any of the tumour samples. SLC22A8/OAT3 is an established high affinity transporter of E-3-S in the kidney and the choroid plexus, and given its absent expression in the tumours and limited expression in the cell lines appears unlikely to play a role in E-3-S uptake in breast cancer. SLC22A7/OAT2 was also not expressed in either of the cell lines, making it unlikely to be of significance in breast cancer. 
The differential expression pattern between the cell lines and human tissues is likely to represent in no small part the heterogeneity of breast cancers, but nevertheless all four tumour samples consistently expressed transporters that both cell lines did not, and vice versa, suggesting some inherent differences between the in vitro cell culture models and fresh invasive ductal carcinoma. One of the challenges with interpreting the human tissue data is the fact that all four patients were receiving endocrine therapy for various periods prior to tumour excision, and this introduces the possibility that the transporter profile may have been influenced by the treatment.

Ideally any tissue samples would have had no prior exposure to treatment, but the difficulties in accessing fresh human tissue are widely acknowledged. Nevertheless, further work to characterise the inducibility of transporter expression using a range of clinically relevant endocrine therapies and anticancer drugs could help in mapping out any changes to the overall transporter profile in breast cancer tissues following drug treatment, which itself may be important in ascertaining response to different treatment types, including the development of drug resistance.

One observation in this study, and a potential limitation, was that in some cases there were discrepancies between the sq-PCR and qPCR results. The exact reason for this is unclear but could be due to differences in chemistries between the two techniques, differences in primers and probes used, or the fact that they rely on different detection methods, with standard PCR measuring the accumulated PCR product at the end of the process and real-time PCR allowing for detection in each amplification phase of the cycle.

Another possibility for discrepancies between the two techniques is the detection of different gene variants. Although these have not been specifically investigated here, the presence of variants has been identified previously. For SLCO1B3, for example, "liver-type" and "cancer-type" gene variants are known to exist (41), and it has been reported that the cancer-type variant of SLCO1B3 was detected in colon, pancreatic and ovarian cancers $(42,43)$. Whether or not this variant, or variants of other SLC members, exist in breast cancer cells, remains to be investigated.

\section{Conclusion}

Breast cancer cells express a panel of SLCs capable of transporting a range of steroid hormones and anticancer drugs, which could implicate their role in $\mathrm{BC}$ pathogenesis and response to treatment. The differential expression patterns across the two cell lines and human tissue samples almost certainly reflect the known heterogeneity of breast cancer, and also reflects the difficulty in treating breast cancer as a single disease entity, but mapping out the expression patterns as comprehensively as possible will help in understanding more about the possible role of transporters in disease pathogenesis or in identifying potential targets for treatment. With more complete mapping of transporter profiles, certain profile 'signatures' particular to certain tumour types may become apparent, and this could one day serve as a prognostic tool.

\section{Abbreviations}


SLC

Solute Carrier

BC

Breast Cancer

$\mathrm{ABC}$

ATP-Binding Cassette

MDR

Multidrug resistance

OATP

Organic anion transporting peptide

OCT

Organic cation transporter

OAT

Organic anion Transporter

OCTN

organic carnitine/zwitterion transporters

ER

Estrogen receptor

PR

Progesterone receptor

HER2

Human epidermal growth factor receptor 2

E-3-S

Estrone-3-Sulfate

\section{Declarations}

Ethics approval and consent to participate: Health Research Authority. North East - Newcastle \& North Tyneside Research Ethics Committee. REC Reference 17/NE/0361

Consent for publication: Not applicable

Availability of data and materials: The datasets used and/or analysed during the current study are available from the corresponding author on reasonable request.

Competing interests: The authors declare that they have no competing interests

Funding: This work was supported by the Women's Cancer Detection Society, Gateshead, UK. Grant number: $\mathrm{BH} 175048$.

Authors' contributions: RS generation of experimental data, data analysis and preparation of manuscript. AM and SL planning of experiments, data analysis and preparation of manuscript. All authors read and 
approved the final manuscript.

\section{Acknowledgements:}

Thanks to Dr Ilse Pienaar, Sussex University, UK, Professor Matthew Wright, Newcastle University, UK and Dr Alastair Greystoke, Newcastle University, UK for cell lines and tissues used as positive controls for PCR experiments.

\section{References}

1. Hanahan D, Weinberg Robert A. Hallmarks of Cancer: The Next Generation. Cell. 2011;144(5):64674.

2. Leonessa F, Clarke R. ATP binding cassette transporters and drug resistance in breast cancer. Endocrine-related Cancer. 2003;10(1):43-73.

3. Doyle LA, Yang W, Abruzzo LV, Krogmann T, Gao Y, Rishi AK, et al. A multidrug resistance transporter from human MCF-7 breast cancer cells. Proc Natl Acad Sci USA. 1998;95(26):15665-70.

4. Volk EL, Farley KM, Wu Y, Li F, Robey RW, Schneider E. Overexpression of wild-type breast cancer resistance protein mediates methotrexate resistance. Cancer research. 2002;62(17):5035-40.

5. Riordan JR, Deuchars K, Kartner N, Alon N, Trent J, Ling V. Amplification of P-glycoprotein genes in multidrug-resistant mammalian cell lines. Nature. 1985;316(6031):817-9.

6. Munoz M, Henderson M, Haber M, Norris M. Role of the MRP1/ABCC1 multidrug transporter protein in cancer. IUBMB Life. 2007;59(12):752-7.

7. Schaller $L$, Lauschke VM. The genetic landscape of the human solute carrier (SLC) transporter superfamily. Human genetics. 2019;138(11-12):1359-77.

8. Obaidat A, Roth $M$, Hagenbuch $B$. The expression and function of organic anion transporting polypeptides in normal tissues and in cancer. Annu Rev Pharmacol Toxicol. 2012;52:135-51.

9. Roth M, Obaidat A, Hagenbuch B. OATPs. OATs and OCTs: the organic anion and cation transporters of the SLCO and SLC22A gene superfamilies. Br J Pharmacol. 2012;165(5):1260-87.

10. Colas C, Ung PM, Schlessinger A. SLC Transporters: Structure, Function, and Drug Discovery. MedChemComm. 2016;7(6):1069-81.

11. Sutherland R, Meeson A, Lowes S. Solute transporters and malignancy: establishing the role of uptake transporters in breast cancer and breast cancer metastasis. Cancer Metastasis Rev. 2020;39(3):919-32.

12. Koepsell H. The SLC22 family with transporters of organic cations, anions and zwitterions. Mol Aspects Med. 2013;34(2-3):413-35.

13. Anzai N, Kanai Y, Endou H. Organic anion transporter family: current knowledge. J Pharmacol Sci. 2006;100(5):411-26.

14. Nakanishi T, Tamai I. Solute carrier transporters as targets for drug delivery and pharmacological intervention for chemotherapy. Journal of pharmaceutical sciences. 2011;100(9):3731-50. 
15. Nigam SK, Bush KT, Martovetsky G, Ahn SY, Liu HC, Richard E, et al. The organic anion transporter (OAT) family: a systems biology perspective. Physiological reviews. 2015;95(1):83-123.

16. Hagenbuch B. Cellular entry of thyroid hormones by organic anion transporting polypeptides. Best practice research Clinical endocrinology metabolism. 2007;21(2):209-21.

17. Stieger B, Hagenbuch B. Organic anion-transporting polypeptides. Curr Top Membr. 2014;73:205-32.

18. Thakkar N, Lockhart AC, Lee W. Role of Organic Anion-Transporting Polypeptides (OATPs) in Cancer Therapy. AAPS J. 2015;17(3):535-45.

19. Banerjee N, Allen C, Bendayan R. Differential role of organic anion-transporting polypeptides in estrone-3-sulphate uptake by breast epithelial cells and breast cancer cells. J Pharmacol Exp Ther. 2012;342(2):510-9.

20. Dai X, Xiang L, Li T, Bai Z. Cancer Hallmarks, Biomarkers and Breast Cancer Molecular Subtypes. J Cancer. 2016;7(10):1281-94.

21. Volk C. OCTs, OATs, and OCTNs: structure and function of the polyspecific organic ion transporters of the SLC22 family. Wiley Interdisciplinary Reviews: Membrane Transport Signaling. 2014;3(1):113.

22. Nies AT, Koepsell H, Damme K, Schwab M. Organic cation transporters (OCTs, MATEs), in vitro and in vivo evidence for the importance in drug therapy. Handbook of experimental pharmacology. 2011(201):105-67.

23. Nigam SK. The SLC22 Transporter Family: A Paradigm for the Impact of Drug Transporters on Metabolic Pathways, Signaling, and Disease. Annu Rev Pharmacol Toxicol. 2018;58:663-87.

24. Douglass S, Meeson AP, Overbeck-Zubrzycka D, Brain JG, Bennett MR, Lamb CA, et al. Breast cancer metastasis: demonstration that FOXP3 regulates CXCR4 expression and the response to CXCL12. J Pathol. 2014;234(1):74-85.

25. Mahkamova K, Latar N, Aspinall S, Meeson A. Hypoxia Increases Thyroid Cancer Stem Cell-Enriched Side Population. World journal of surgery. 2018;42(2):350-7.

26. Britton KM, Eyre R, Harvey IJ, Stemke-Hale K, Browell D, Lennard TWJ, et al. Breast cancer, side population cells and ABCG2 expression. Cancer letters. 2012;323(1):97-105.

27. Wlcek K, Svoboda M, Thalhammer T, Sellner F, Krupitza G, Jaeger W. Altered expression of organic anion transporter polypeptide (OATP) genes in human breast carcinoma. Cancer Biol Ther. 2008;7(9):1450-5.

28. Miki Y, Suzuki T, Kitada K, Yabuki N, Shibuya R, Moriya T, et al. Expression of the steroid and xenobiotic receptor and its possible target gene, organic anion transporting polypeptide-A, in human breast carcinoma. Cancer research. 2006;66(1):535-42.

29. Pizzagalli F, Varga Z, Huber RD, Folkers G, Meier PJ, St-Pierre MV. Identification of steroid sulfate transport processes in the human mammary gland. J Clin Endocrinol Metab. 2003;88(8):3902-12.

30. Cai H, Zhang Y, Han TK, Everett RS, Thakker DR. Cation-selective transporters are critical to the AMPK-mediated antiproliferative effects of metformin in human breast cancer cells. International 
journal of cancer. 2016;138(9):2281-92.

31. Okabe M, Szakács G, Reimers MA, Suzuki T, Hall MD, Abe T, et al. Profiling SLCO and SLC22 genes in the NCl-60 cancer cell lines to identify drug uptake transporters. Mol Cancer Ther. 2008;7(9):308191.

32. Wang C, Uray IP, Mazumdar A, Mayer JA, Brown PH. SLC22A5/OCTN2 expression in breast cancer is induced by estrogen via a novel intronic estrogen-response element (ERE). Breast cancer research treatment. 2012;134(1):101-15.

33. Iusuf D, Hendrikx JJ, van Esch A, van de Steeg E, Wagenaar E, Rosing H, et al. Human OATP1B1, OATP1B3 and OATP1A2 can mediate the in vivo uptake and clearance of docetaxel. International journal of cancer. 2015;136(1):225-33.

34. van de Steeg E, van Esch A, Wagenaar E, Kenworthy KE, Schinkel AH. Influence of human OATP1B1, OATP1B3, and OATP1A2 on the pharmacokinetics of methotrexate and paclitaxel in humanized transgenic mice. Clin Cancer Res. 2013;19(4):821-32.

35. König J, Seithel A, Gradhand U, Fromm MF. Pharmacogenomics of human OATP transporters. Naunyn Schmiedebergs Arch Pharmacol. 2006;372(6):432-43.

36. Kindla J, Rau TT, Jung R, Fasching PA, Strick R, Stoehr R, et al. Expression and localization of the uptake transporters OATP2B1, OATP3A1 and OATP5A1 in non-malignant and malignant breast tissue. Cancer Biol Ther. 2011;11(6):584-91.

37. Bleasby K, Castle JC, Roberts CJ, Cheng C, Bailey WJ, Sina JF, et al. Expression profiles of 50 xenobiotic transporter genes in humans and pre-clinical species: a resource for investigations into drug disposition. Xenobiotica. 2006;36(10-11):963-88.

38. Pressler H, Sissung TM, Venzon D, Price DK, Figg WD. Expression of OATP family members in hormone-related cancers: potential markers of progression. PloS one. 2011;6(5):e20372.

39. Muto M, Onogawa T, Suzuki T, Ishida T, Rikiyama T, Katayose Y, et al. Human liver-specific organic anion transporter-2 is a potent prognostic factor for human breast carcinoma. Cancer Sci. 2007;98(10):1570-6.

40. Olszewski-Hamilton U, Svoboda M, Thalhammer T, Buxhofer-Ausch V, Geissler K, Hamilton G. Organic Anion Transporting Polypeptide 5A1 (OATP5A1) in Small Cell Lung Cancer (SCLC) Cells: Possible Involvement in Chemoresistance to Satraplatin. Biomark Cancer. 2011;3:31-40.

41. Alam K, Farasyn T, Ding K, Yue W. Characterization of Liver- and Cancer-type-Organic Anion Transporting Polypeptide (OATP) 1B3 Messenger RNA Expression in Normal and Cancerous Human Tissues. Drug metabolism letters. 2018;12(1):24-32.

42. Thakkar N, Kim K, Jang ER, Han S, Kim K, Kim D, et al. A cancer-specific variant of the SLC01B3 gene encodes a novel human organic anion transporting polypeptide 1B3 (OATP1B3) localized mainly in the cytoplasm of colon and pancreatic cancer cells. Mol Pharm. 2013;10(1):406-16.

43. Svoboda M, Mungenast F, Gleiss A, Vergote I, Vanderstichele A, Sehouli J, et al. Clinical Significance of Organic Anion Transporting Polypeptide Gene Expression in High-Grade Serous Ovarian Cancer. Front Pharmacol. 2018;9:842. 
Figures

(A)

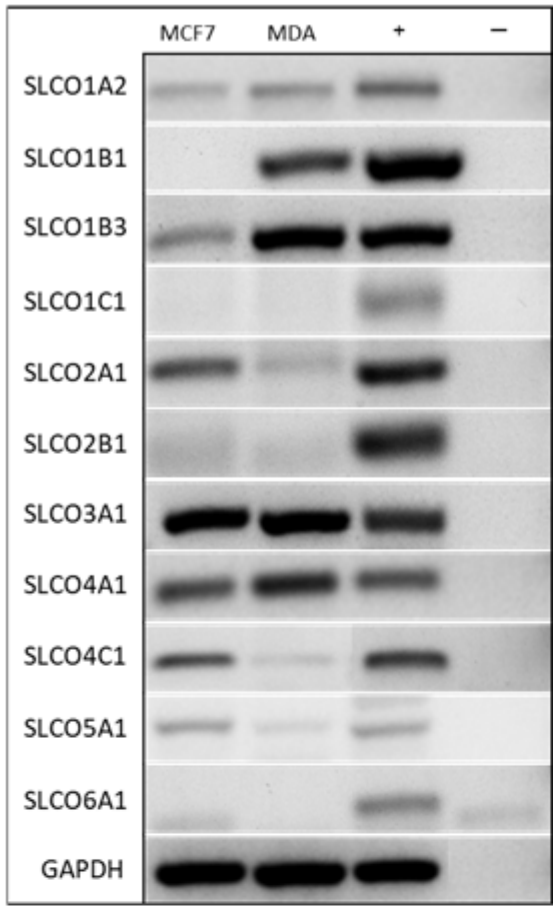

(B)
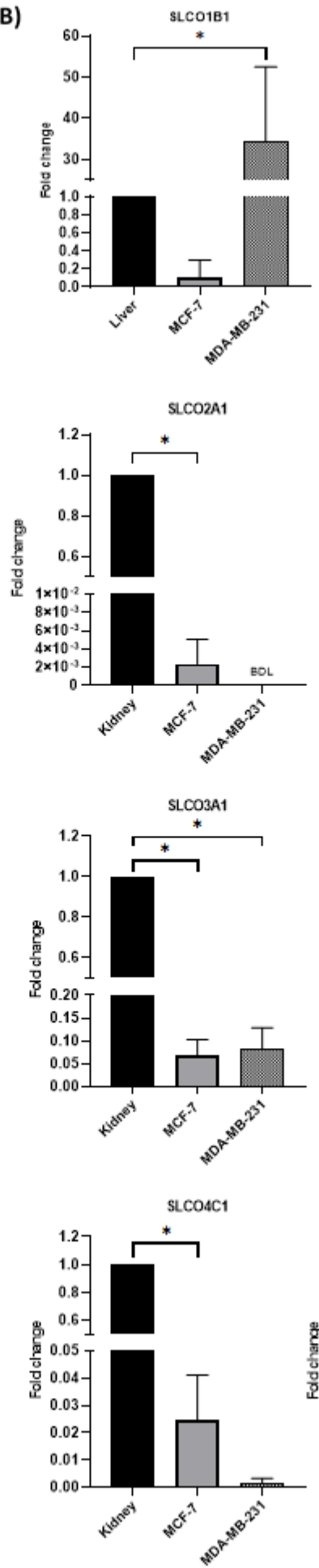
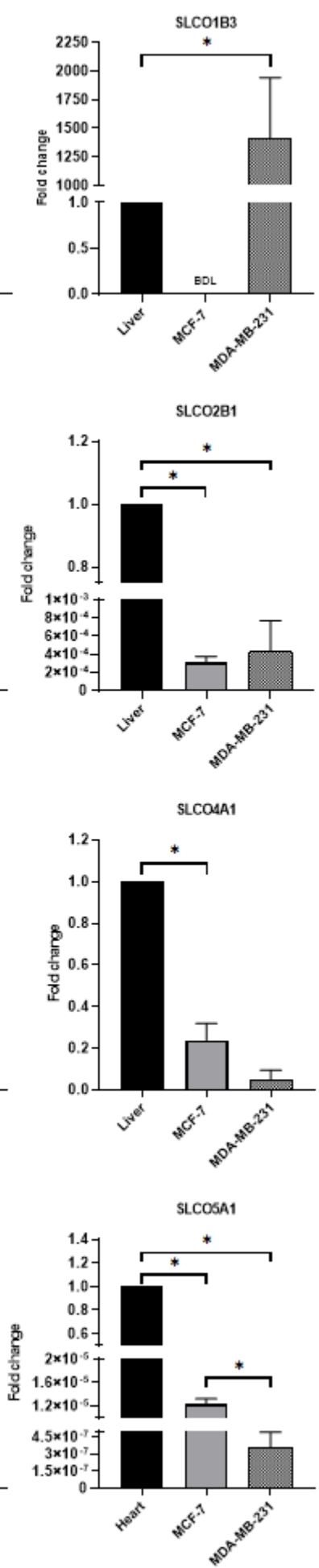

Figure 1

Expression of SLCO transporters in MCF-7 and MDA-MB-231 cell lines. (A) Semi-quantitative PCR results show expression of SLCO transporter genes in MCF-7 and MDA-MB-231 cell lines. SLC01A2, SLC01B1, SLC01B3, SLC01C1, SLC02A1, SLC02B1, SLC03A1, SLC04A1, SLC04C1, SLC05A1 and SLC06A1 (+) 
positive control, (-) negative control. GAPDH was used as a loading control for each experiment.

Representative of three independent experiments. (B) Relative mRNA expression of SLCO Transporters in MCF-7 and MDA-MB-231 cell lines determined via qPCR. Relative expression of SLC01B1 (MCF-7 = 0.11, MDA-MB-231 = 34.26), SLC01B3 (MCF-7 = BDL, MDA-MB-231 = 1417.72), SLCO2A1 (MCF-7 = 0.002, MDA-MB-231 = BDL), SLCO2B1 (MCF-7 = 0.0003, MDA-MB-231 = 0.0004), SLC03A1 (MCF-7 = 0.07, MDAMB-231 = 0.08), SLCO4A1 (MCF-7 = 0.24, MDA-MB-231 = 0.05), SLC04C1 (MCF-7 = 0.02, MDA-MB-231 = 0.001 ) and SLC05A1 (MCF-7 $=0.00001$, MDA-MB-231 $=0.0000004)$ is shown. Results are expressed as fold change relative to a calibrator sample and normalised to the housekeeping gene $\beta$-actin. Data represented as mean \pm SD of three independent experiments. Statistical significance was calculated using a one-sample and/or independent samples t-test $\left({ }^{*} p<0.05\right)$. BLD = below detection limit. 
(A)

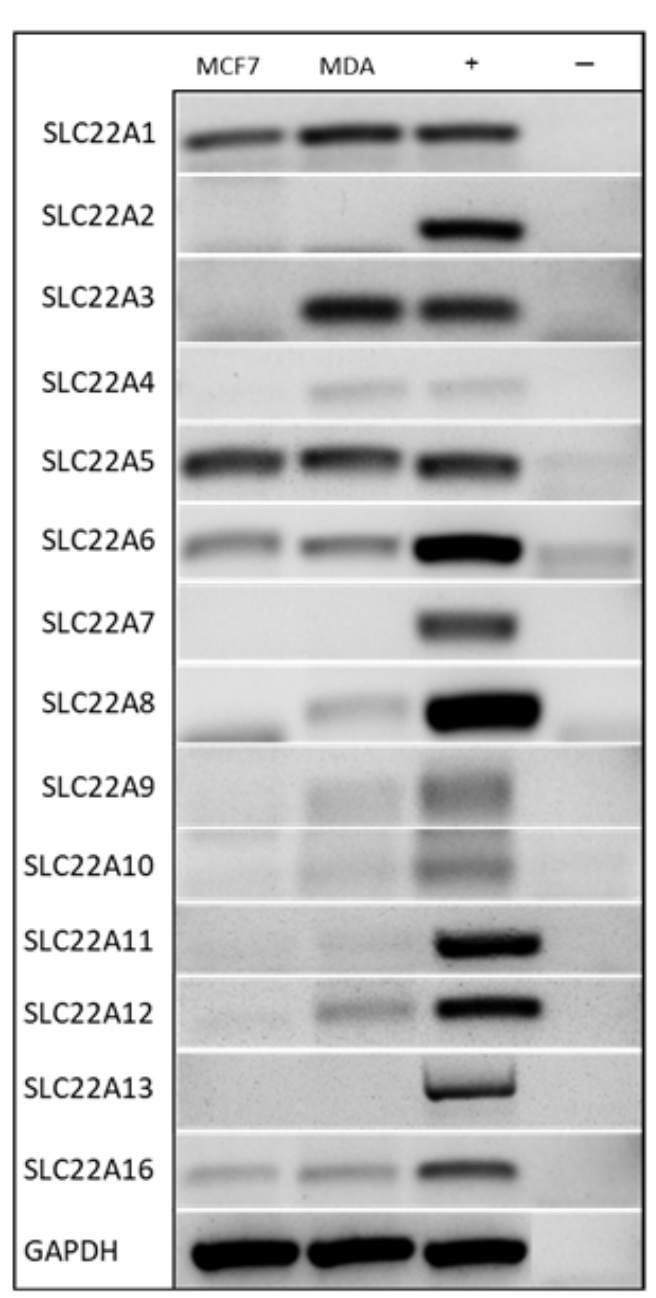

(B)
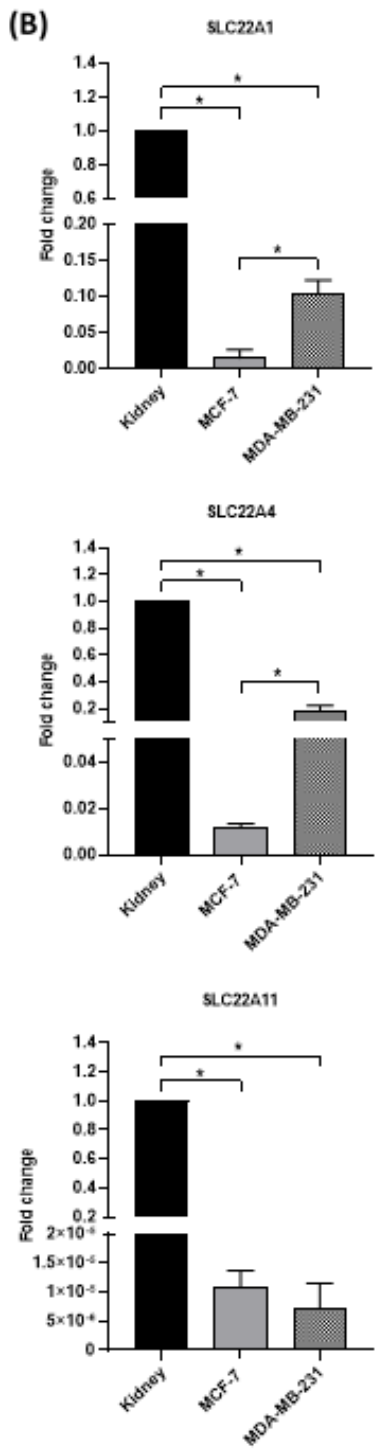
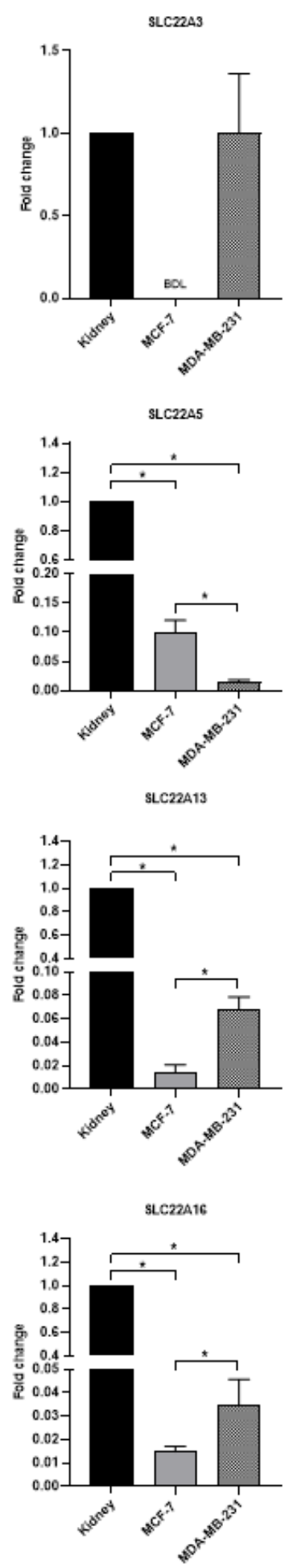

\section{Figure 2}

Expression of SLC22 transporters in MCF-7 and MDA-MB-231 cell lines. (A) Semi-quantitative PCR results show expression of SLC22 transporter genes in MCF-7 and MDA-MB-231 cell lines. SLC22A1, SLC22A2, SLC22A3, SLC22A4, SLC22A5, SLC22A6, SLC22A7, SLC22A8, SLC22A9, SLC22A10, SLC22A11, SLC22A12, SLC22A13 and SLC22A16. (+) positive control, (-) negative control. GAPDH was used as a loading control for each experiment. Representative of three independent experiments. (B) Relative mRNA 
expression of SLC22 Transporters in MCF-7 and MDA-MB-231 cell lines determined via qPCR. Relative expression of SLC22A1 (MCF-7 = 0.01, MDA-MB-231 = 0.10), SLC22A3 (MCF-7 = BDL, MDA-MB-231 = 1.00), SLC22A4 (MCF-7 = 0.01, MDA-MB-231 = 0.19), SLC22A5 (MCF-7 = 0.1, MDA-MB-231 0.01), SLC22A11 (MCF-7 = 0.00001, MDA-MB-231 = 0.000007), SLC22A13 (MCF-7 = 0.01, MDA-MB-231 0.07) and SLC22A16 (MCF-7 $=0.01$, MDA-MB-231 $=0.04$ ) is shown. Results are expressed as fold change relative to a calibrator sample and normalised to the housekeeping gene $\beta$-actin. Data represented as mean \pm SD of three independent experiments. Statistical significance was calculated using a one-sample or independent samples t-test $\left({ }^{*} \mathrm{p}<0.05\right)$. BLD = below detection limit.

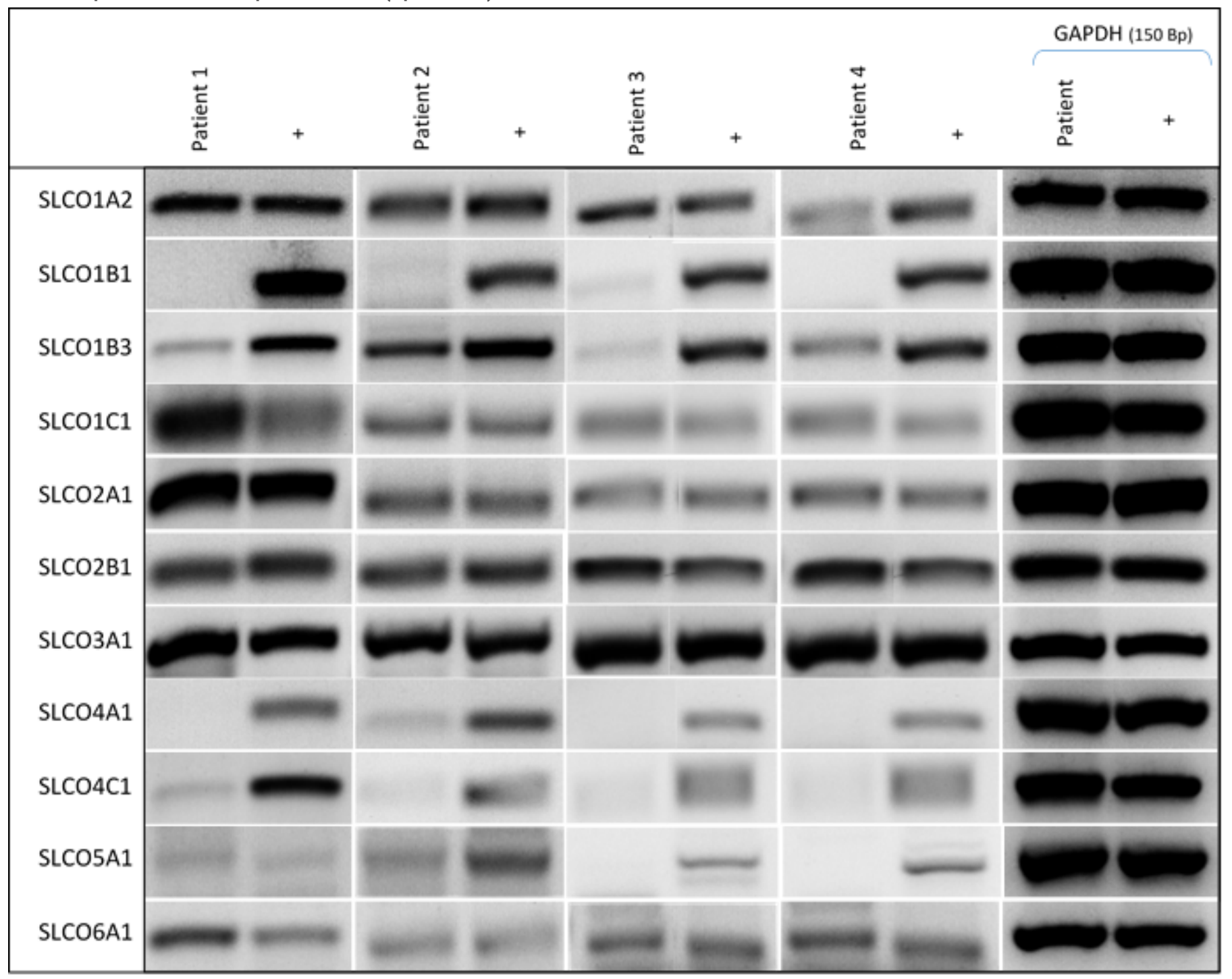

\section{Figure 3}

Semi-quantitative PCR results show expression of SLCO transporter genes in four patient-derived breast tumour samples. (+) positive control, GAPDH was used as a loading control for all experiment. A representative example of GAPDH expression in included in the figure. 

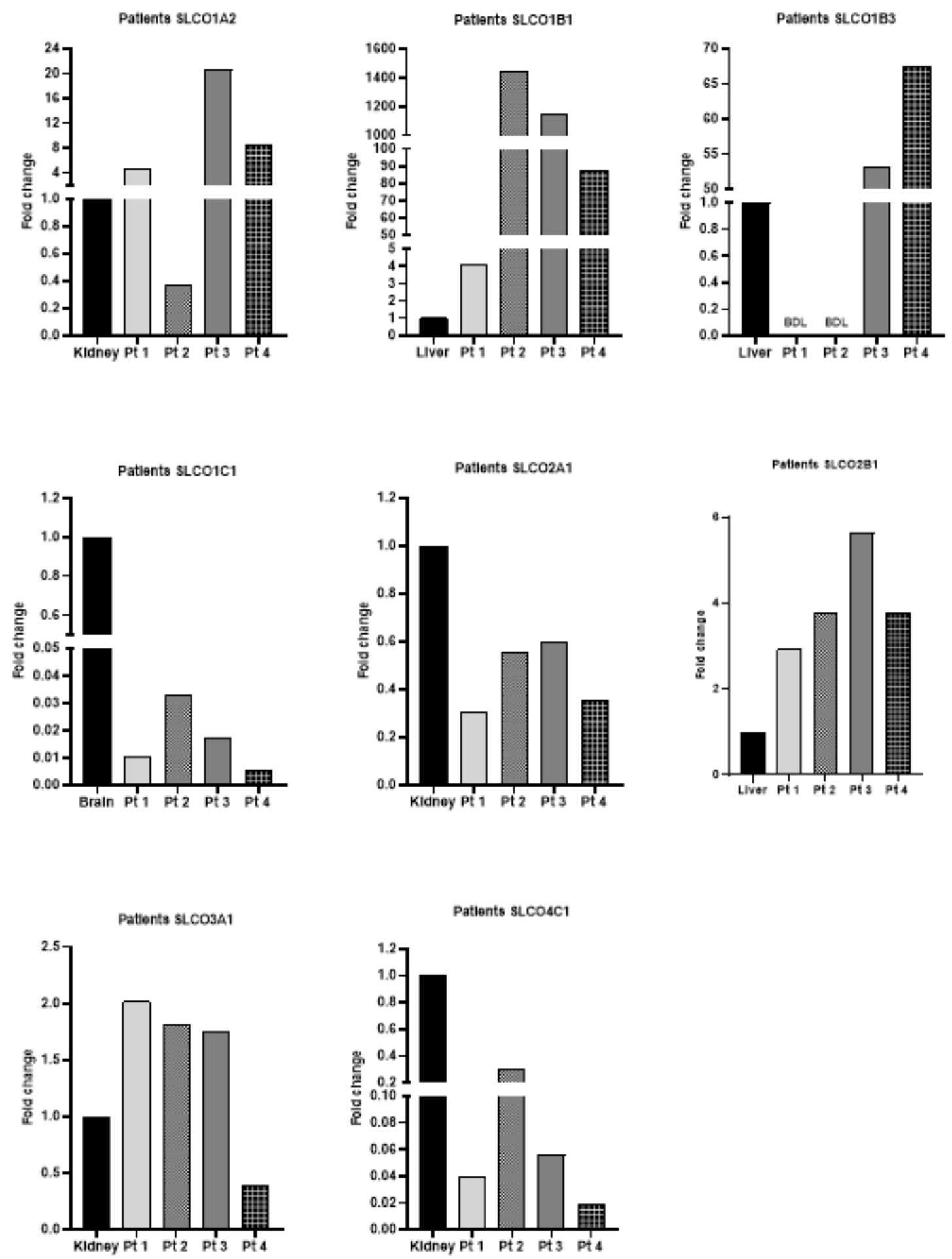

Figure 4

Relative mRNA expression of SLCO Transporters in patient breast cancer derived tumours via qPCR. Results are expressed as fold change relative to a calibrator and normalised to the housekeeping gene $\beta$ actin. 


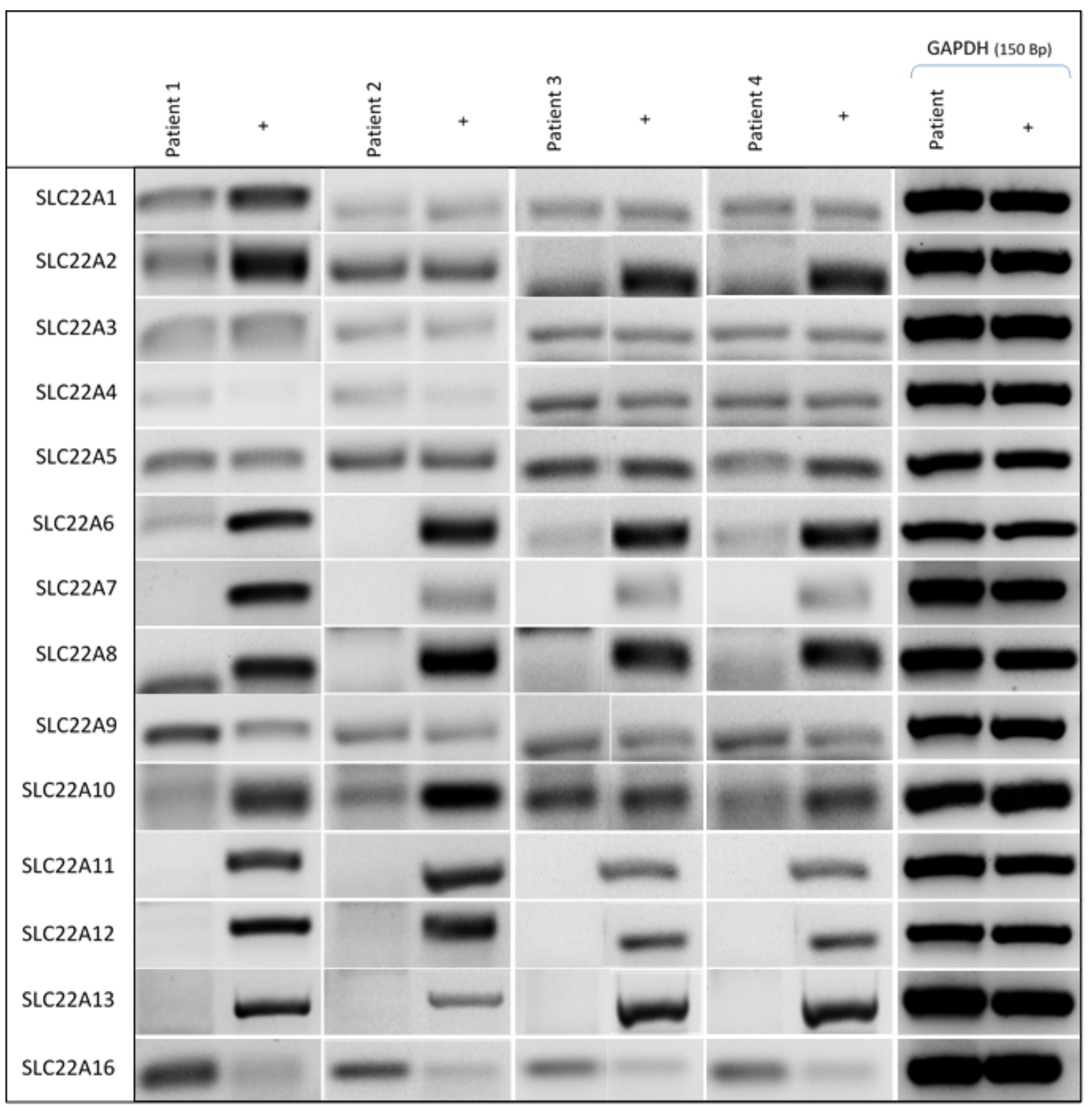

\section{Figure 5}

Expression of SLCO transporter genes in breast cancer samples from four patients using sq-PCR. (+) positive control, GAPDH was used as a loading control for all experiments. A representative example of GAPDH expression in included in the figure. 

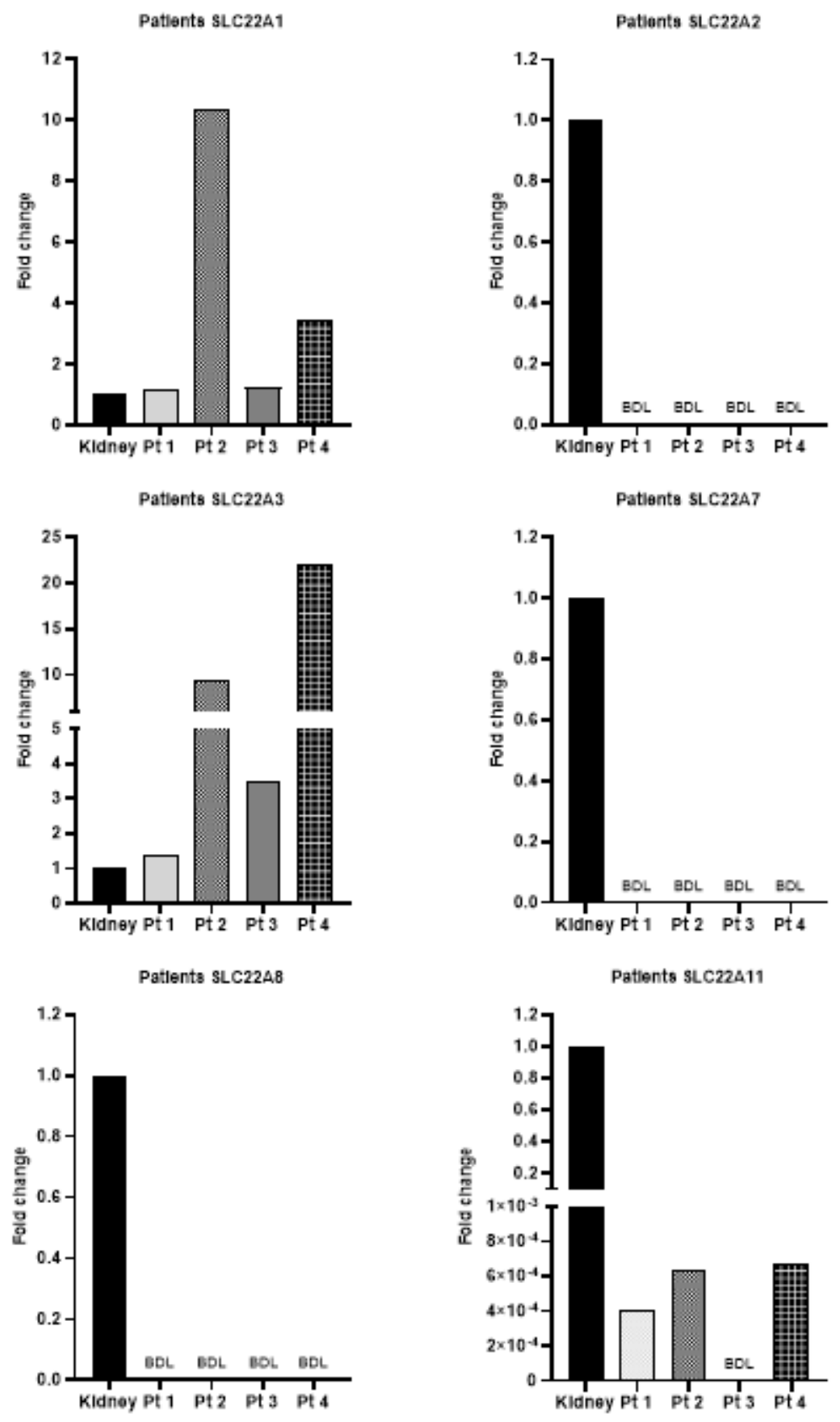

Figure 6

Relative mRNA expression of selected SLC22 transporters in patient breast cancer tumour samples using qPCR. Results are expressed as fold change relative to a calibrator and normalised to the housekeeping gene $\beta$-actin.

\section{Supplementary Files}

This is a list of supplementary files associated with this preprint. Click to download. 
- Additionalfile1.docx

Page 22/22 